•研究报告・

\title{
不同纬度高蔊菜的交配系统和繁殖保障
}

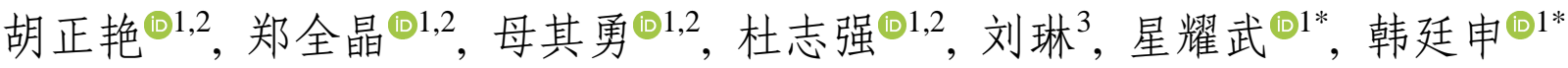

1. 中国科学院西双版纳热带植物园热带森林生态学重点实验室, 云南预腊 666303; 2. 中国科学院大学, 北京 100049; 3. 香格里拉高山植 物园, 云南迪庆 674400

摘要: 高山植物的繁殖适应策略一直是进化生态学研究的热点, 但是目前仍缺乏对繁殖适应性状的量化研究。本研究以横断 山特有十字花科植物高蔊菜(Rorippa elata)为研究材料, 结合野外同质园控制实验, 在居群水平探讨了其繁殖适应策略。结果 表明, 高草菜的繁殖能力与纬度呈负相关, 而越冬存活率与纬度呈正相关。不同交配方式结实率的显著性差异分析表明, 高 蔊菜具有混合交配系统，其中自交为主型和中间型的株系各占45.5\%和39.4\%; 不同株系具有不同程度的繁殖保障现象，自交 为主型最高(0.163), 中间型最低(0.011)。本研究表明高草菜在居群水平的繁殖和存活之间存在沿纬度梯度的权衡变化, 以自 交为主的混合交配系统为其提供不同程度的繁殖保障。

关键词: 适应性; 同质园; 横断山; 权衡; 交配系统; 结实率

胡正艳, 郑全晶, 母其勇, 杜志强, 刘琳, 星耀武, 韩廷申 (2021) 不同纬度高蔊菜的交配系统和繁殖保障. 生物多样性, 29, 712-721. doi: 10.17520/biods.2021056.

Hu ZY, Zheng QJ, Mu QY, Du ZQ, Liu L, Xing YW, Han TS (2021) The mating system and reproductive assurance of Rorippa elata (Brassicaceae) across latitude. Biodiversity Science, 29, 712-721. doi: 10.17520/biods.2021056.

\section{The mating system and reproductive assurance of Rorippa elata (Brassicaceae) across latitude}

Zhengyan $\mathrm{Hu}^{\circledR 1,2}$, Quanjing Zheng ${ }^{\circledR 1,2}$, Qiyong $\mathrm{Mu}^{\circledR 1,2}$, Zhiqiang $\mathrm{Du}^{\circledR 1,2}$, Lin Liu ${ }^{3}$, Yaowu Xing ${ }^{\circledR 1^{*}}$, Ting-Shen $\operatorname{Han}^{(1 *}$

1 CAS Key Laboratory of Tropical Forest Ecology, Xishuangbanna Tropical Botanical Garden, Chinese Academy of Sciences, Mengla, Yunnan 666303

2 University of Chinese Academy of Sciences, Beijing 100049

3 Shangri-La Alpine Botanical Garden, Diqing, Yunnan 674400

\section{ABSTRACT}

Aim: The strategies of reproductive adaptation for alpine plants have long been a hot topic in evolutionary ecology, but there is still a lack of quantitative measures for reproductive traits under natural conditions. Here, we investigated reproductive adaptation of Rorippa elata, an endemic Brassicaceae species in one of the world's temperate biodiversity hotspots, the Hengduan Mountains (HDM).

Methods: We established a field-based common garden experiment at Shangri-La Alpine Botanical Garden in Yunnan. During 2019-2020, we transplanted a total of 633 R. elata plants for 150 accessions across latitudes and performed manipulative field experiments. First, we collected several life history-related traits of $R$. elata, including germination rate, flowering time, over-winter survival rate, and pollination-relevant characteristics. We then carried out a set of four experimental treatments to characterize natural variation of the mating system in $R$. elata, including (1) strict selfing rate by bagging inflorescences before flowering; (2) common outcrossing rate by emasculation and open pollination; (3) strict outcrossing rate by emasculation and artificial pollination among accessions; and (4) natural reproductive rate under open pollination. For each treatment, we counted seed setting rate per fruit per accession and used this information to assign a mating system type for each $R$. elata accession. Furthermore, we also quantified the extents of pollen limitation and reproductive assurance.

收稿日期: 2021-02-09; 接受日期: 2021-04-08

基金项目: 国家自然科学基金(31800177; U1802242)、中国科学院青年创新促进会(2020391)和中国科学院“西部之光”人才培养计划

* 共同通讯作者 Co-authors for correspondence. E-mail: ywxing@xtbg.org.cn; hantingshen@xtbg.ac.cn 
Results: Our results showed that the main visitors of $R$. elata flowers were insects of Syrphidae, Halictus, Musca domestica, and Calliphoridae. Among these, insects of Syrphidae visited flowers most frequently and insects of Calliphoridae had the longest staying time per visiting. According to life history observations in the common garden, we demonstrated that $R$. elata plants germinated in early spring followed by a long period of reproductive growth throughout the synchronization of both high temperature and precipitation in HDM. They then entered into dormancy with living sprouts belowground during cold and dry winter. Correlation analysis of latitude with seed setting rate and over-winter survival rate showed that latitude was negatively correlated with the reproductive ability but positively correlated with the over-winter survival rate of $R$. elata. Comparative analysis of seed setting rates among experimental treatments found that $R$. elata possessed a mixed mating system, with selfing dominant and intermediate types accounting for $45.5 \%$ and $39.4 \%$ of accessions, respectively. Furthermore, most $R$. elata accessions showed signs of reproductive assurance, but to different extents, with the highest in selfing dominant accessions (0.163) and the lowest in intermediate accessions (0.011).

Conclusions: Our findings reveal the reproductive strategies of $R$. elata at the population level and underscore a trade-off between reproduction and survival along a latitudinal gradient. The mixed mating system with variable reproductive assurance likely plays an important role in the stability of reproduction. Collectively, our results highlight the important contributions of reproductive strategies for alpine plants during their adaptation to mountain environments.

Key words: adaptation; common garden; Hengduan Mountains; trade-off; mating system; seed setting rate

高山生物区(alpine biome)是唯一在全球广泛分 布的生物区, 也是地球上极端环境分布最集中和受 气候变化威胁最严重的地区之一 (Körner, 2020; Testolin et al, 2020; Wang T et al, 2021)。高山植物 (alpine plant)是地理分布中心位于高山生物区的植 物总称(Körner, 2003)。在长期极端环境的选择压力 下, 高山植物进化出了多样的适应性策略(Peng et al, 2014; Sun et al, 2014), 尤其是在繁殖方面(彭德 力等, 2012; Barrett, 2014; Barrett \& Harder, 2017; Tong et al, 2020)。比如, 在传粉者稀缺时, 植物会通 过花冠弯曲(Sun et al, 2005)、花瓣闭合时雄莣移动 (Xiong et al, 2013)、异型花 柱向同型花柱转换(Wang XJ et al, 2021)等方式实现自交繁殖; 许多高山植物 在有性繁殖的同时, 亦具有克隆繁殖能力(Körner, 2003; 郝楠等, 2016)等。但是目前尚不清楚同一高 山物种不同居群间的繁殖方式是否存在差异以及 不同繁殖方式的比例是否不同(张婵等, 2020)。

作为一系列适应性性状的主要代表, 存活率和 繁殖能力可客观地反映植物适应能力的高低, 因而 成为衡量植物适合度最常用的指标之一(Wadgymar et al, 2017; Friedman, 2020; Lundgren \& Des Marais, 2020)。研究表明, 高山植物为了应对复杂多变的高 山环境, 往往会将有限的资源在存活和繁殖之间进 行不均等分配, 该过程称为分配权衡 (allocation trade-off) (Weiner, 2004; Sun et al, 2014; 张婵等, 2020)。如果植物将较多的资源分配在提高存活率上,
那么对繁殖方面的投入可能就会相应地减少(Wenk \& Falster, 2015)。以往的研究多集中在探讨高山植 物在有性繁殖和克隆繁殖之间(Bengtsson \& Ceplitis, 2000; 张婵等, 2020)以及交配系统内部(自交与异 交的主次地位)的权衡(Peng et al, 2012; Barrett, 2014; Nasrallah, 2017), 较少关注多年生高山植物繁殖能 力与存活率之间的权衡。

交配系统的演化是高山植物适应性进化过程 中的重要环节。按交配对象的来源可分为自交、异 交和混合交配系统三种(予茜等, 2008; Moeller et al, 2017)。自交作为一种繁殖保障策略, 常发生在传粉 受限的情况下(Busch \& Delph, 2012); 当缓解近亲 繁殖压力的成本超过自交优势的时候, 植物会倾向 于提高异交率(Whitehead et al, 2018); 混合交配系 统是介于专性自交和专性异交之间的交配方式, 即 同时拥有自交和异交两种交配能力。混合交配系统 频繁出现在被子植物中(Goodwillie et al, 2005; Munoz et al, 2016), 其中自交繁殖保障的程度在不 同物种或居群间具有环境依赖性 (Kalisz et al, 2004)。由于相关野外观察与控制实验的开展具有一 定的难度, 导致目前对混合交配系统的类型(如自 交和异交各自的占比)及其生态或进化意义尚缺乏 深入研究。利用同质园实验来研究混合交配系统, 可以直接衡量和比较不同居群植物的繁殖相关表 型, 是开展植物繁殖适应性研究的理想方法 (de Villemereuil et al, 2016; Tong et al, 2020)。 
横断山区拥有全球最丰富的温带高山植物多 样性(Ding et al, 2020), 是开展高山植物繁殖适应性 进化研究的天然实验室(Peng et al, 2014; Sun et al, 2017; Tong et al, 2020)。据统计, 横断山区大约有 $88 \%$ 的有花植物为两性花, 可以自交的物种占比高 达97.1\% (Peng et al, 2014)。高草菜(Rorippa elata) 是横断山特有的十字花科物种, 分布在海拔 2,000-4,500 m的地区, 为研究高山植物的繁殖适应 性进化提供了理想材料。本研究利用野外同质园控 制实验平台开展高蔊菜的繁殖生态学相关实验, 拟 回答以下 3 个科学问题: (1)高澣菜在居群水平是否 存在繁殖与存活之间的分配权衡? (2)高草菜是否具 有混合交配系统? 如果有, 其混合交配系统的类型 有哪些? (3)高蔊菜不同居群是否存在繁殖保障? 如 果有, 其繁殖保障的程度是否存在差异?

\section{1 材料与方法}

\section{1 实验材料}

\subsection{1 野外采样及温室植株培养}

2017-2019年, 在横断山区以居群为单位采集
高蔊菜的成熟种子(图1A、B), 每个居群的单棵植株 为一个株系, 单独收集不同株系的种子。将野外采 集的种子用 $1 / 2$ MS培养基(Duchefa Biochemie, The Netherlands)萌发。每个株系选择20-25粒种子置于 $2 \mathrm{~mL}$ 离心管, 加入蒸馏水浸泡4-5 h; 在超净工作台 用蒸馏水和 $70 \%$ 酒精交叉清洗6次, 之后将其分散 铺在倒有 $1 / 2 \mathrm{MS}$ 固体培养基的培养血中, 吸干多余 水分后用封口膜(PARAfILM-996, USA)封口; 24个 培养血为一组用铝䈃纸遮光处理, 置于 $4^{\circ} \mathrm{C}$ 进行为 期 2 周的均一化处理; 随后在人工气候室 $(16 \mathrm{~h}$ 光照 $+8 \mathrm{~h}$ 黑暗, $20^{\circ} \mathrm{C}, 60 \%$ 空气相对湿度)中萌发 1 个星 期。萌发的种子以每个培养血6棵植株作为重复, 利 用R程序(https://github.com/Ting-Shen/Rorippa-polyp loids-in-HDM)对每一株植物进行随机排序和编号 后转移至装有营养土和蛭石(按1：3配置)的育苗盘 中, 在人工气候室中连续培养 2 个月; 之后将植株 移栽至同质园中。

\subsection{2 同质园建立}

同质园位于云南省迪庆藏族自治州香格里拉 市的香格里拉高山植物园 $\left(27.91^{\circ} \mathrm{N}, 99.64^{\circ} \mathrm{E}\right.$, 海拔

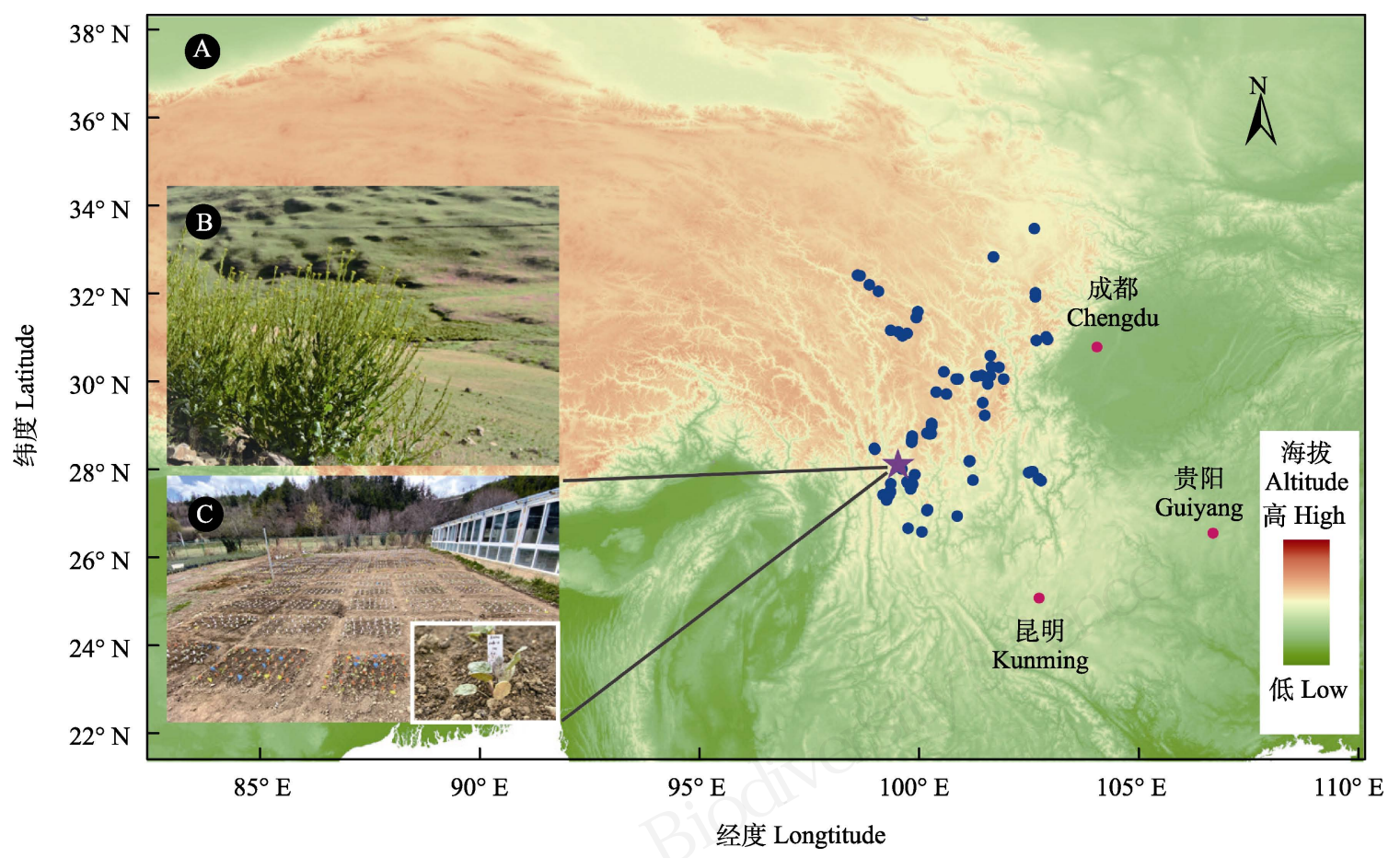

图1 高蔊菜野外采样点及同质园位置。A: 高蔊菜野外采样点(蓝色点)及同质园位置(紫色星号标示); B: 高蔊菜野外居群照 片; C: 同质园全景照及移栽的高蔊菜植株。

Fig. 1 Sample sites of Rorippa elata natural populations and the common garden. A, Sampled R. elata populations (blue dots) and the location of common garden (purple star); B, Photo for the natural population of R. elata; C, Common garden and transplanted seedlings of $R$. elata. 
3,300 m)。同质园及其周边是高蔊菜分布的典型生 境, 园内亦分布有高澣菜的天然居群。2019年4月, 将633株高蔊菜幼苗移栽至同质园, 包括77个居群 150 个株系。以 5 株 $\times 5$ 株为一个样方移栽, 植株间 距为 $15 \mathrm{~cm}$, 样方间垄宽为 $30 \mathrm{~cm}$ (图1 C)。

\section{2 实验方法}

\subsection{1 访花者和生活史观测}

采用样方法对高蔊菜访花者进行监测。选取一 定面积的样方, 记录样方内开放的花朵总数, 用计 时器记录不同访花者每小时进入样方的次数及单 次访花时长。计算每类访花者的单次观测访花频率 及单次平均访花时长, 访花频率 $=$ 访花次数总和 $/($ 样方内开放花朵总数 $\times 1 \mathrm{~h})$, 最后计算每种访花 者的平均访花频率。单次平均访花时长 $=$ 访花时 长总和/访花次数。

2019年4月至2020年8月, 定期记录高草菜各阶 段的生长情况, 并对其生活史过程进行观测。观测 对象包括移栽至同质园的高蔊菜植株, 以及香格里 拉高山植物园内的高蔊菜天然居群。

\subsection{2 实验处理}

对移栽至同质园的高草菜开花植株开展如下 4 种实验处理: (1)开放授粉: 对移栽植株不做任何处 理, 使其自然授粉和结实。(2)人工套袋: 选取主花 序轴的侧枝或非主花序轴, 去除已经开放的花朵后, 用可以阻隔外来花粉的育种袋套袋, 使其自然授粉 和结实; 处理 $20 \mathrm{~d}$ 后将育种袋拆除, 使果荚在自然 状态下发育至成熟。单棵植株选取3个以上分枝进 行人工套袋处理。(3)人工去雄开放授粉: 在晴天选 取主花序轴上3-5朵完好的、颜色变黄且快要开放 的花苞, 用事先进行 70\%酒精消毒的镊子去除所有 花药, 同时去除同一花序轴上所有已开放的花朵和 顶端分生组织, 然后使其自然授粉和结实。每棵植 株共选取至少 8 朵小花进行人工去雄和开放授粉处 理。(4)人工去雄杂交: 选取主花序轴上未开放的、 花苍颜色变黄的3-5朵花作为花粉受体 (母本), 于实 验前一天去雄后套袋(方法同上); 次日或第三日上 午10:00-12:00期间, 选取来自不同居群的植株作为 花粉供体(父本), 将其花药开裂的花朵倒扣在已去 雄的母本花朵上, 使花药与柱头充分接触, 以能观 察到有花粉留在柱头上为准; 授粉完成后再次套上 育种袋, 并做好标记; 处理7天后将育种袋拆除, 使 果荚在自然状态下发育至成熟。

\section{3 表型统计}

\subsection{1 结实率}

在实验结束时, 单个收取成熟果荚并对其进行 镜检。在体视镜(Motic, SMZ-171-BL)下, 用镊子剥 离单个果荚的外壳, 对其中的总胚珠数目 $\left(N_{\text {ovule }}\right)$ 和 饱满种子数目 $\left(N_{\text {seed }}\right)$ 分别进行计数, 并计算其结实 率( $\mathrm{SS}=N_{\text {seed }} / N_{\text {ovule }}$ )。本研究所用到的基本统计量为 高蔊菜每个株系在各实验处理下的平均结实率, 具 体定义如下:

开放授粉结实率 $\left(\mathrm{SS}_{\mathrm{open}}\right)$ 指自然状态下的结实 率。每棵植株至少统计 15 个果荚, 分别于单棵植株 主花序轴的顶端、中间与下部各取 5 个。

自交结实率( $\mathrm{SS}_{\text {selfing }}$ )指经过人工套袋处理后的 结实率。每棵植株统计 5 个果荚, 每个株系的植株重 复数 $\geq 3$ 。

去雄开放授粉结实率 $\left(\mathrm{SS}_{\mathrm{out}}\right)$ 指在人工去雄后自 然状态下的结实率。每个株系的植株重复数 $\geq 3$ 。

杂交结实率 $\left(\mathrm{SS}_{\mathrm{hyb}}\right)$ 指在人工去雄后人工授粉处 理下的结实率。每个株系的植株重复数 $\geq 3$ 。

\subsection{2 繁殖性状}

(1)交配系统类型。根据不同实验处理下结实率 的高低, 对每个株系的交配系统类型进行界定。若 同时可以进行自交和异交结实 $\left(\mathrm{SS}_{\text {selfing }}>0, \mathrm{SS}_{\text {out }}>\right.$ 0 ), 即为混合交配系统。以往的研究基于异交结实 率来划分植物的交配系统。其中, 混合交配系统的 判定标准是植物的异交结实率介于 0.2-0.8之间 (Goodwillie et al, 2005)。该混合交配系统的划分范 围较为笼统和宽泛, 且忽略了混合交配系统内部可 能存在的变异。因此, 本研究利用统计学分析方法 将混合交配系统划分为自交为主型 $\left(\mathrm{SS}_{\text {selfing }} \approx \mathrm{SS}_{\text {open, }}\right.$, $\left.\mathrm{SS}_{\text {selfing }}>>\mathrm{SS}_{\text {out }}\right) 、$ 中间型 $\left(\mathrm{SS}_{\text {selfing }} \approx \mathrm{SS}_{\text {open }} \approx \mathrm{SS}_{\text {out }}\right)$ 和 互补型 $\left(\mathrm{SS}_{\text {open }}>>\mathrm{SS}_{\text {selfing, }}, \mathrm{SS}_{\text {open }}>>\mathrm{SS}_{\text {out }}\right) 3$ 种。

(2)花粉限制。由于落在柱头上的花粉不足而导 致的结实受限现象(Ashman et al, 2004)称为花粉限 制 (pollen limitation, PL)。本研究参考Ashman等 (2004)的研究方法, 以杂交和开放授粉结实率的差 值对此进行评估:

$$
\mathrm{PL}=\mathrm{SS}_{\mathrm{hyb}}-\mathrm{SS}_{\text {open }}
$$

若PL $<0$, 表明此株系在自然状态下的结实未受到 花粉数目的限制; 若PL $>0$, 且在统计上 $\mathrm{SS}_{\mathrm{hyb}}$ 显著 高于 $S_{\text {open }}(t$-test, $P<0.05)$, 则说明此株系存在花 粉限制。 
(3)繁殖保障。在配子或传粉者稀少的情况下, 自花授粉可以维持繁殖(予茜等, 2008), 称为生殖或 繁殖保障(reproductive assurance, RA)。本研究参考 Busch和Delph (2012)对繁殖保障的量化方法, 以去 雄开放授粉和开放授粉结实率的比值来反映繁殖 保障的有无及其高低:

$$
\mathrm{RA}=1-\mathrm{SS}_{\text {out }} / \mathrm{SS}_{\text {open }}
$$

若RA $<0$, 说明不存在繁殖保障; 反之, 若RA $>0$, 则表明存在繁殖保障现象; RA的变化可代表繁殖保 障的变异程度。

\subsection{3 存活率}

存活率指越冬存活率。以居群为单位, 次年 (2020年)存活的植株总数占 2019年移栽植株的比 例。仅统计植株总数 $\geq 10$ 的居群。

\section{4 数据分析}

通过 R 代码包 raster v.2.6-6 从 WorldClim (http://worldclim.org/) 获取同质园所在地的月平均 温度和降水数据, 结合高澣菜各生长阶段对应绘图 描述其生活史过程。使用简单线性回归分析方法, 对高澣菜天然居群的纬度与繁殖力 (由开放授粉结 实率衡量)及存活率的相关性进行分析; 采用单因 素方差分析(one-way ANOVA)和最小显著性差异检 验(LSD test)分析比较高薂菜各株系不同实验处理 下的结实率, 并据此结果划分交配系统类型; 运用 单因素方差分析和Tukey HSD test检测高草菜不同
交配系统类型的株系是否存在花粉限制现象。以上 分析均在R v.3.6.2 (R Core Team, 2019)中完成。

\section{结果}

\section{1 访花者和生活史}

同质园内高蔊菜的访花者及其访花行为的监 测结果表明, 高草菜的主要访花者为双翅目食蚜蝇 科、蝇科家蝇 (Musca domestica) 和丽蝇科及膜翅目 隧蜂科隧蜂属(Halictus) (图2)。食蚜蝇科的昆虫访 花频率最高, 为 0.097 次每朵每小时; 其次为隧蜂属 的昆虫, 访花频率为 0.050 次每朵每小时, 但二者的 单次访花平均时长均较短 (分别为 $127.857 \mathrm{~s}$ 和 $80.721 \mathrm{~s})$ 。单次访花平均时间最长的为丽蝇科昆虫 (531.500 s), 其次是家蝇(302.167 s), 但其单位时间 内访花频次均较低, 分别为 0.030 和 0.044 次每朵每 小时。

生活史观测结果表明：3月至4月中旬，高蔊菜 萌发并进行营养生长(图3I); 4月中下旬至6月为花期, 此时随着温度升高, 传粉昆虫活动日趋频繁, 植株 进行生殖生长(图3II); 7-9 月, 正值横断山区的雨热 同期季节，高澣菜果荚逐渐成熟并开裂，散落到地 上的部分种子会萌发并长出幼苗, 多年生株系的植 株继续进行营养生长, 并在莲座叶的叶腋处产生嫩 芽(图3III)；10月至次年2月，地上部分枯黄，地下部 分及叶腋处嫩芽被积雪覆盖并进行休眠(图3IV)。

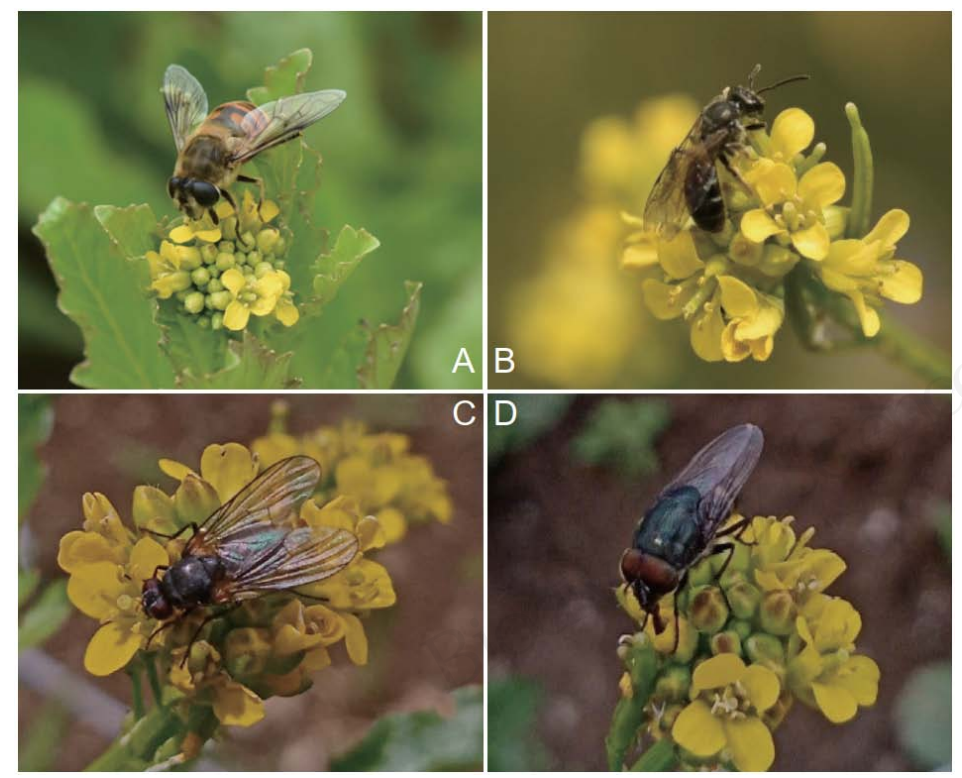

图 2 高蔊菜主要访花者。A: 食蚜蝇科; B: 隧蜂属; C: 家蝇; D: 丽蝇科。

Fig. 2 Main visitors of Rorippa elata. A, Syrphidae; B, Halictus; C, Musca domestica; D, Calliphoridae. 


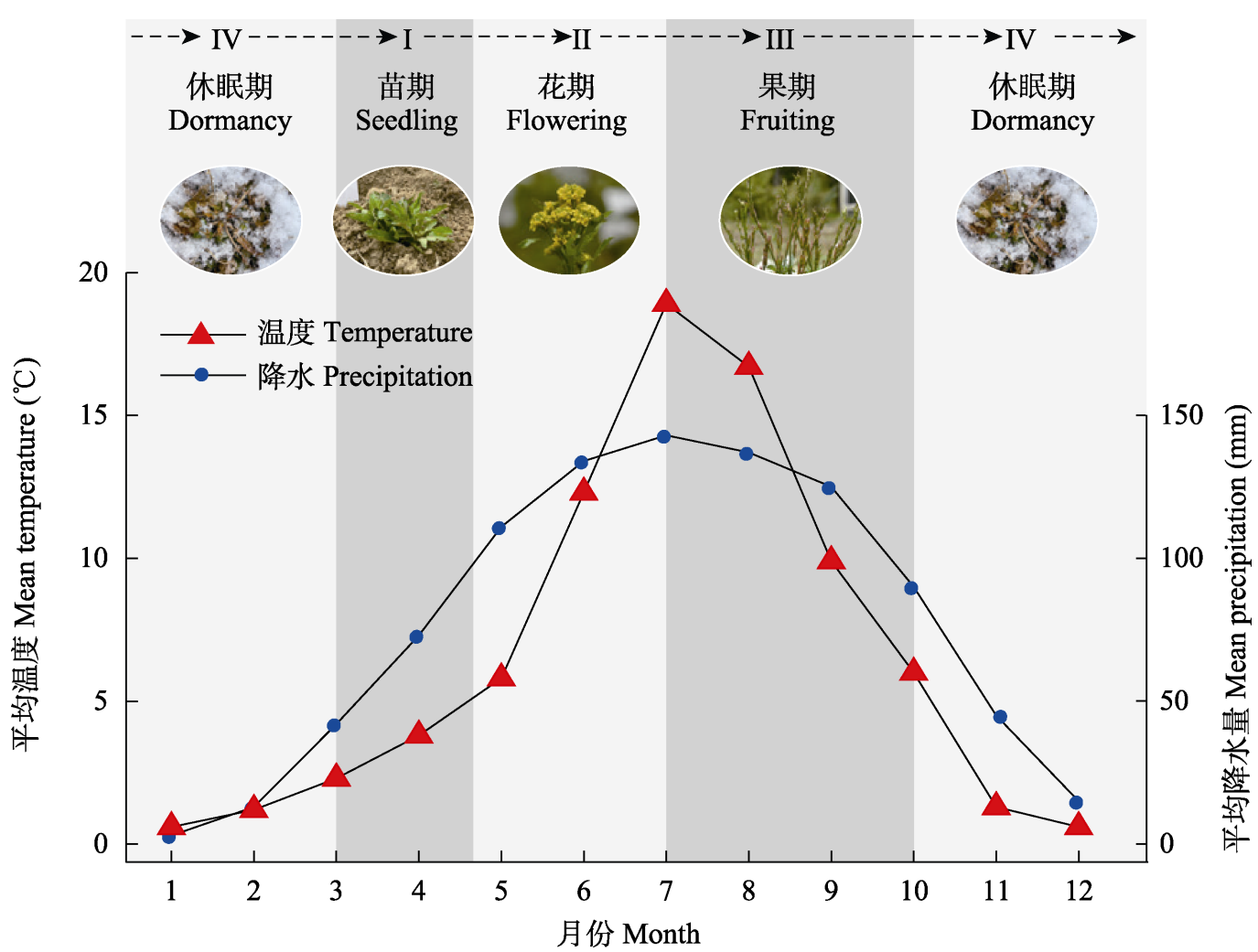

图3＼cjkstart高蔊菜生活史图示及同质园内每月温度和降水变化

Fig. 3 Life history of Rorippa elata and temperature or precipitation variation per month in the common garden
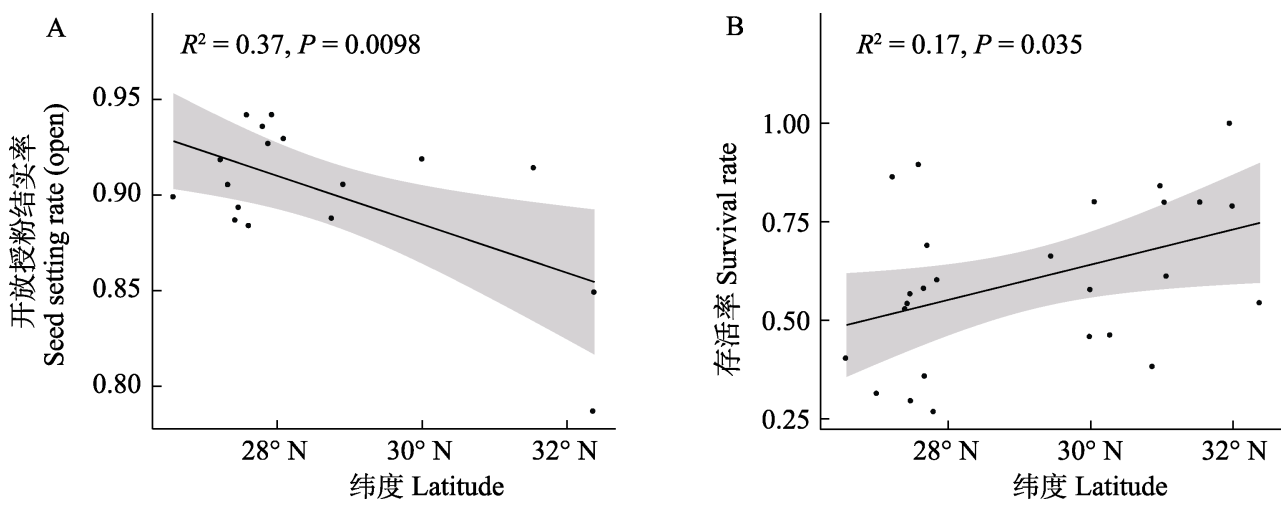

图4 高蔊菜开放授粉结实率(A)及越冬存活率(B)与纬度的相关性分析

Fig. 4 Correlation analysis of seed setting rate (open) (A) and over-winter survival rate (B) with latitude of Rorippa elata populations

\section{2 表型与纬度相关性分析}

随着纬度升高, 高蔊菜的开放授粉结实率显著 降低(图4A, $R^{2}=0.37, P=0.0098$ ), 而越冬存活率则 显著升高(图4B, $\left.R^{2}=0.17, P=0.035\right)$, 表明高蔊菜 在繁殖和存活之间存在沿纬度梯度的权衡。

\section{3 结实率分析}

\subsection{1 交配系统类型}

对33个高蔊菜株系在不同实验处理下的结实 率的统计分析发现, 高蔊菜具有混合交配系统, 即
自花授粉和异花授粉均能成功产生种子。对交配系 统类型进行进一步划分发现, 高蔊菜具有自交为主 型、中间型和互补型3种混合交配系统类型, 其中自 交为主型株系占所有统计株系的45.5\% (图5A), 中 间型株系的占比为39.4\% (图5B), 而互补型株系占 比最低(15.1\%) (图5C)。

\subsection{2 花粉限制}

花粉限制分析结果表明，仅有3个株系检测到 花粉限制现象 $(\mathrm{PL}>0, P<0.05)$, 分别是较弱花粉 

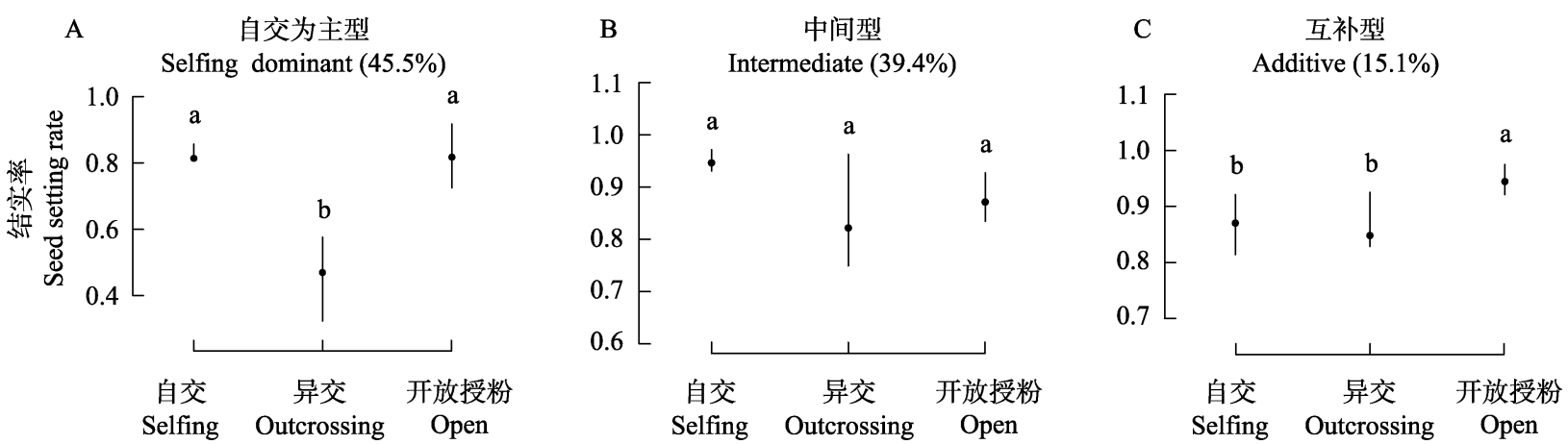

图5 高蔊菜的交配系统类型。不同字母表示不同实验处理的结实率之间差异显著 $(\boldsymbol{P}<0.05)$ 。

Fig. 5 Mating system type of Rorippa elata. Different letters indicate significant differences among seed setting rate of different experimental treatments $(P<0.05)$.

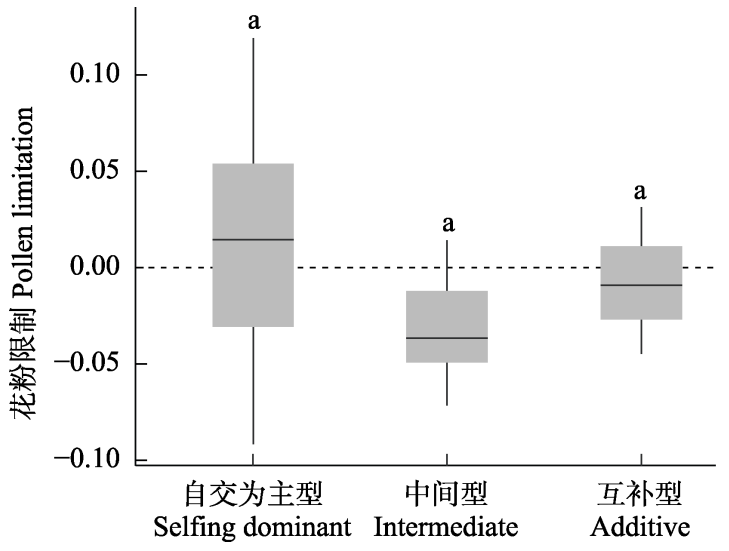

图6 不同类型交配系统的花粉限制分析。不同字母表示不 同交配系统的花粉限制差异显著 $(P<0.05)$ 。

Fig. 6 Pollen limitation of different types mating system. Different letters indicate significant differences among mating system types of different pollen limitation $(P<0.05)$.

限制的RO531-1 (PL = 0.067, $P=0.018)$ 和RO468-6 $(\mathrm{PL}=0.073, P=0.006)$, 及花粉限制较强的 RO537-2 (PL = 0.119, $P=0.007$ ) (附录1)。分别对3 种混合交配系统类型的花粉限制进行统计发现(图 6), 自交为主型具有较高的花粉限制水平, 但不同 混合交配系统类型间在花粉限制上的差异不显著。

\subsection{3 自交繁殖保障}

繁殖保障的量化研究结果显示(图7), 高蔊菜 不同株系具有不同的繁殖保障现象。15例自交为主 型株系普遍具有繁殖保障现象, 且平均程度较高 $(\mathrm{RA}=0.163 \pm 0.016)$, 其中仅有 1 例未检测到 (RO466-5, RA = -0.051)。13例中间型株系中, 超过 一半的株系(7例)具有较弱的繁殖保障现象 $(\mathrm{RA}=$ $0.061 \pm 0.001)$, 剩余株系均未检测到, 导致中间型 株系的平均繁殖保障程度近乎为 $0(\mathrm{RA}=0.011 \pm$ 0.005)。所有5例互补型株系均具有繁殖保障现象,
整体平均程度中等 $(\mathrm{RA}=0.075 \pm 0.002)$, 但其中 RO463-2的繁殖保障程度较弱 $(\mathrm{RA}=0.001)$ 。在平均 繁殖保障程度方面, 自交为主型的显著高于中间型 的 $(P=0.0002)$; 互补型低于自交为主型、高于中间 型, 但差异均不显著 $(P=0.063)$ 。

\section{3 讨论}

高山植物的生殖生长受到温度、降水和光周期 等环境因子的调控, 例如高山早熟禾(Poa alpina)的 开花需要春化作用和光周期共同调控 (Körner, 2003)。本研究基于两年同质园实验观察, 发现高澣 菜的生殖生长处于横断山区雨热同期时间段内(图 3), 良好的水热条件有利于植株繁殖器官的发育。 此外, 高蔊菜具有部分种子在当年萌发并进行营养 生长的现象(图3), 支持Miller和Geddes (2004)提出 的“高山多花植物的种子通常在秋季萌发并完成形 态建成”的结论。

高山植物的分配权衡策略反映了其对高山环 境变化的响应。与以往研究不同, 本研究对高山植 物高澣菜的生存与繁殖两个重要适应性性状进行 了衡量(图4)。结果表明, 高蔊菜为适应横断山区多 变的环境, 在繁殖能力和存活率之间存在沿纬度梯 度的分配权衡(图4), 相似结果也发现于分布在青藏 高原的马先蒿属(Pedicularis)植物的研究中(Guo et $\mathrm{al}, 2012)$ 。与单个气候因子相比, 纬度信息作为反映 横断山区气候环境变化的综合性指标, 可以代表温 度、降水和光照强度等诸多环境因子的梯度变化。 植物通过沿纬度梯度的分配权衡, 一方面可以缓和 随纬度升高而愈加严苛的高山环境对繁殖发育的 限制，例如极端低温或干旱、传粉者减少或传粉活 


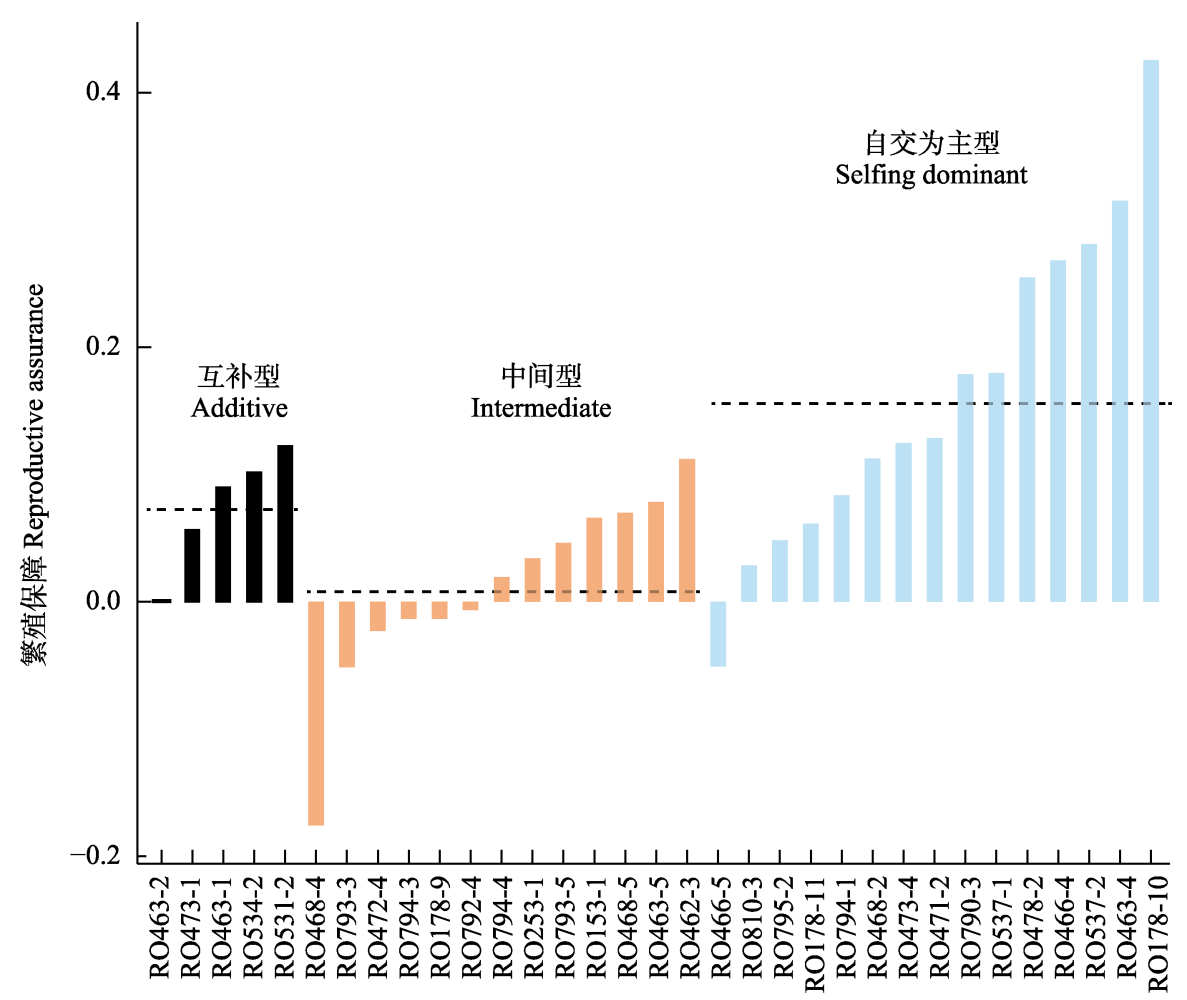

株系 Accession

图7 高蔊菜各株系和交配系统类型的繁殖保障分析

Fig. 7 Reproductive assurance across Rorippa elata accessions and mating system types

动受限等; 另一方面, 也可以利用更多的时间合成 和积累能量, 最终实现繁殖上的成功。

混合交配系统是高山植物普遍采用的繁殖适 应策略(张婵等, 2020)。本研究综合不同观测和实验 处理结果, 认为高草菜具有混合交配系统, 其中异 交可能由食蚜蝇、隧蜂等访花或传粉昆虫介导(图 2)。彭德力等(2012)认为, 自交亲和可能是植物应对 高山环境下传粉限制的适应机制。在传粉者活动减 弱时, 专性异交繁殖的高山植物将会面临较高的灭 绝风险, 而长期专性自交繁殖则可能导致近交衰退 等不利后果。因此, 混合交配系统被认为是高山植 物成功繁殖的双重保障, 且多存在于虫媒传粉植物 中(Goodwillie et al, 2010)。相较于其他植物交配系 统相关的工作, 本研究对混合交配系统类型进行了 更细致的划分, 发现高蔊菜不同株系的混合交配系 统类型不同，其中以自交为主型和中间型为主，以 互补型为辅(图5)。进一步的研究表明, 上述现象并 非受到花粉数目限制的直接影响(图6), 而更可能是 为适应高山极端多变环境所采取的繁殖适应策略 (图7)。

混合交配系统可由多种途径维持, 包括传粉者
介导的同株异花受精(Devaux et al, 2014)和滞后自 交(Brys et al, 2016; Goodwillie \& Weber, 2018)等, 但是自交繁殖保障在其中发挥的作用尚较少涉及 (Elle \& Carney, 2003)。本研究发现，高草菜的不同 株系具有不同程度的繁殖保障现象, 其中自交为主 型显著高于中间型，自交和异交程度相近的中间型 具有最低的繁殖保障程度(图7)。在混合交配系统中, 自花授粉机制为高山植物提供了传递优势与繁殖 保障, 由此驱动和维持自交的进化 (Foxe et al, 2009)。但另一方面，自交会增加基因的纯合性，降 低遗传多样性, 并使有害的隐性基因暴露出来, 进 而引起近交衰退(Charlesworth \& Willis, 2009)或种 子折损(Herlihy \& Eckert, 2002; Layman et al, 2017) 等不利后果。除此之外, 相较于无性繁殖, 有性繁 殖需要分配更多的能量用于繁殖器官的发育(张婵 等, 2020)。基于此我们推断，自交带来的繁殖保障 优势可能需要与上述自交劣势或资源投资相抵消 或权衡。因此, 本研究在高蔊菜居群中检测到的差 异性繁殖保障现象, 可能暗示着高澣菜在自交繁殖 保障与近交衰退之间存在某种权衡，从而维持了混 合交配系统在高山植物适应性进化中的作用。 
本研究揭示了高澣菜的混合交配系统类型及 差异性繁殖保障对混合交配系统的维持作用, 但是 混合交配系统内部异交与自交的发生时序、两者所 产生后代的适应能力以及混合交配系统的长期进 化意义等, 需要进一步深入研究。

\section{ORCID}

胡正艳 (D) https://orcid.org/0000-0002-0691-0238

郑全晶 (D) https://orcid.org/0000-0001-8820-2042

母其勇 (i) https://orcid.org/0000-0002-5976-5724

杜志强 (iD) https://orcid.org/0000-0001-9589-3857

星耀武 (iD https://orcid.org/0000-0001-6709-4492

韩廷申 (iD) https://orcid.org/0000-0002-8612-6581

\section{参考文献}

Ashman TL, Knight TM, Steets JA, Amarasekare P, Burd M, Campbell DR, Dudash MR, Johnston MO, Mazer SJ, Mitchell RJ, Morgan MT, Wilson WG (2004) Pollen limitation of plant reproduction: Ecological and evolutionary causes and consequences. Ecology, 85, 2408-2421.

Barrett SCH (2014) Evolution of mating systems: Outcrossing versus selfing. In: The Princeton Guide to Evolution (eds Losos JB, Baum DA, Futuyma DJ, Hoekstra EH, Lenski RE, Moore AJ, Peichel GL, Schluter D, Whitlock MC), pp. 356-362. Princeton University Press, Princeton.

Barrett SCH, Harder LD (2017) The ecology of mating and its evolutionary consequences in seed plants. Annual Review of Ecology, Evolution, and Systematics, 48, 135-157.

Bengtsson BO, Ceplitis A (2000) The balance between sexual and asexual reproduction in plants living in variable environments. Journal of Evolutionary Biology, 13, 415-422.

Brys R, van Cauwenberghe J, Jacquemyn H (2016) The importance of autonomous selfing in preventing hybridization in three closely related plant species. Journal of Ecology, 104, 601-610.

Busch JW, Delph LF (2012) The relative importance of reproductive assurance and automatic selection as hypotheses for the evolution of self-fertilization. Annals of Botany, 109, 553-562.

Charlesworth D, Willis JH (2009) The genetics of inbreeding depression. Nature Reviews Genetics, 10, 783-796.

Devaux C, Lande R, Porcher E (2014) Pollination ecology and inbreeding depression control individual flowering phenologies and mixed mating. Evolution, 68, 3051-3065.

de Villemereuil P, Gaggiotti OE, Mouterde M, Till-Bottraud I (2016) Common garden experiments in the genomic era: New perspectives and opportunities. Heredity, 116, 249-254.

Ding WN, Ree RH, Spicer RA, Xing YW (2020) Ancient orogenic and monsoon-driven assembly of the world's richest temperate alpine flora. Science, 369, 578-581.
Elle E, Carney R (2003) Reproductive assurance varies with flower size in Collinsia parviflora (Scrophulariaceae). American Journal of Botany, 90, 888-896.

Foxe JP, Slotte T, Stahl EA, Neuffer B, Hurka H, Wright SI (2009) Recent speciation associated with the evolution of selfing in Capsella. Proceedings of the National Academy of Sciences, USA, 106, 5241-5245.

Friedman J (2020) The evolution of annual and perennial plant life histories: Ecological correlates and genetic mechanisms. Annual Review of Ecology, Evolution, and Systematics, 51, 461-481.

Goodwillie C, Weber JJ (2018) The best of both worlds? A review of delayed selfing in flowering plants. American Journal of Botany, 105, 641-655.

Goodwillie C, Kalisz S, Eckert CG (2005) The evolutionary enigma of mixed mating systems in plants: Occurrence, theoretical explanations, and empirical evidence. Annual Review of Ecology, Evolution, and Systematics, 36, 47-79.

Goodwillie C, Sargent RD, Eckert CG, Elle E, Geber MA, Johnston MO, Kalisz S, Moeller DA, Ree RH, Vallejo-Marin M, Winn AA (2010) Correlated evolution of mating system and floral display traits in flowering plants and its implications for the distribution of mating system variation. New Phytologist, 185, 311-321.

Guo H, Weiner J, Mazer SJ, Zhao ZG, Du GZ, Li B (2012) Reproductive allometry in Pedicularis species changes with elevation. Journal of Ecology, 100, 452-458.

Hao N, Su X, Wu Q, Chang LB, Zhang SH, Sun K (2016) Size-dependent of Qinghai-Tibetan Plateau Viola tuberifera (Violaceae) bulbs allocation. Guihaia, 36, 674-678. (in Chinese with English abstract) [郝楠, 苏雪, 吴琼, 常立博, 张世虎, 孙坤 (2016) 青藏高原东缘块茎堇菜鳞茎分配 的个体大小依赖性. 广西植物, 36, 674-678.]

Herlihy CR, Eckert CG (2002) Genetic cost of reproductive assurance in a self-fertilizing plant. Nature, 416, 320-323.

Kalisz S, Vogler DW, Hanley KM (2004) Context-dependent autonomous self-fertilization yields reproductive assurance and mixed mating. Nature, 430, 884-887.

Körner C (2003) Alpine Plant Life: Functional Plant Ecology of High Mountain Ecosystems. Springer, Berlin.

Körner C (2020) Plant adaptations to alpine environments. In: Encyclopedia of the World's Biomes (eds Goldstein MI, DellaSala DA), pp. 355-361. Elsevier, Amsterdam.

Layman NC, Fernando MTR, Herlihy CR, Busch JW (2017) Costs of selfing prevent the spread of a self-compatibility mutation that causes reproductive assurance. Evolution, 71, 884-897.

Lundgren MR, Des Marais DL (2020) Life history variation as a model for understanding trade-offs in plantenvironment interactions. Current Biology, 30, R180-R189.

Miller GR, Geddes C (2004) Seed-setting by alpine gentian (Gentiana nivalis L.). Botanical Journal of Scotland, 56, 85-91.

Moeller DA, Briscoe Runquist RD, Moe AM, Geber MA, 
Goodwillie C, Cheptou PO, Eckert CG, Elle E, Johnston MO, Kalisz S, Ree RH, Sargent RD, Vallejo-Marin M, Winn AA (2017) Global biogeography of mating system variation in seed plants. Ecology Letters, 20, 375-384.

Munoz F, Violle C, Cheptou PO (2016) CSR ecological strategies and plant mating systems: Outcrossing increases with competitiveness but stress-tolerance is related to mixed mating. Oikos, 125, 1296-1303.

Nasrallah JB (2017) Plant mating systems: Self-incompatibility and evolutionary transitions to self-fertility in the mustard family. Current Opinion in Genetics \& Development, 47, 54-60.

Peng DL, Zhang ZQ, Niu Y, Yang Y, Song B, Sun H, Li ZM (2012) Advances in the studies of reproductive strategies of alpine plants. Biodiversity Science, 20,29286 (in Chinese with English abstract) [彭德力, 张志强, 牛洋, 杨 扬, 宋波, 孙航, 李志敏 (2012) 高山植物繁殖策略的研 究进展. 生物多样性, 20, 286-299.]

Peng DL, Zhang ZQ, Xu B, Li ZM, Sun H (2012) Patterns of flower morphology and sexual systems in the subnival belt of the Hengduan Mountains, SW China. Alpine Botany, 122, 65-73.

Peng DL, Ou XK, Xu B, Zhang ZQ, Niu Y, Li ZM, Sun H (2014) Plant sexual systems correlated with morphological traits: Reflecting reproductive strategies of alpine plants. Journal of Systematics and Evolution, 52, 368-377.

R Core Team (2019) R: A Language and Environment for Statistical Computing. $\mathrm{R}$ Foundation for Statistical Computing, Vienna.

Sun H, Niu Y, Chen YS, Song B, Liu CQ, Peng DL, Chen JG, Yang Y (2014) Survival and reproduction of plant species in the Qinghai-Tibet Plateau. Journal of Systematics and Evolution, 52, 378-396.

Sun H, Zhang JW, Deng T, Boufford DE (2017) Origins and evolution of plant diversity in the Hengduan Mountains, China. Plant Diversity, 39, 161-166.

Sun SG, Guo YH, Gituru RW, Huang SQ (2005) Corolla wilting facilitates delayed autonomous self-pollination in Pedicularis dunniana (Orobanchaceae). Plant Systematics and Evolution, 251, 229-237.

Testolin R, Attorre F, Jiménez-Alfaro B (2020) Global distribution and bioclimatic characterization of alpine biomes. Ecography, 43, 779-788.

Tong ZY, Wu LY, Huang SQ (2020) Reproductive strategies of animal-pollinated plants on high mountains: A review of studies from the "Third Pole". Journal of Systematics and Evolution, doi: 10.1111/jse.12680.

Wadgymar SM, Daws SC, Anderson JT (2017) Integrating viability and fecundity selection to illuminate the adaptive nature of genetic clines. Evolution Letters, 1, 26-39.

Wang T, Zhao YT, Xu CY, Ciais P, Liu D, Yang H, Piao SL, Yao TD (2021) Atmospheric dynamic constraints on Tibetan Plateau freshwater under Paris climate targets. Nature Climate Change, 11, 219-225.

Wang XJ, Barrett SCH, Zhong L, Wu ZK, Li DZ, Wang H, Zhou W (2021) The genomic selfing syndrome accompanies the evolutionary breakdown of heterostyly. Molecular Biology and Evolution, 38, 168-180.

Weiner J (2004) Allocation, plasticity and allometry in plants. Perspectives in Plant Ecology, Evolution and Systematics, 6, 207-215.

Wenk EH, Falster DS (2015) Quantifying and understanding reproductive allocation schedules in plants. Ecology and Evolution, 5, 5521-5538.

Whitehead MR, Lanfear R, Mitchell RJ, Karron JD (2018) Plant mating systems often vary widely among populations. Frontiers in Ecology and Evolution, 6, doi: 10.3389/fevo.2018.00038.

Xiong YZ, Fang Q, Huang SQ (2013) Pollinator scarcity drives the shift to delayed selfing in Himalayan mayapple Podophyllum hexandrum (Berberidaceae). AoB Plants, 5, plt037.

Yu Q, Zhang YW, Guo YH (2008) Translation and elucidation of common terms in pollination biology. Journal of Systematics and Evolution, 46, 9602. (in Chinese with English abstract) [予茜, 张彦文, 郭友好 (2008) 传粉生 物学常用术语释译. 植物分类学报, 46, 96-102.]

Zhang C, An YM, Jäschke Y, Wang LL, Zhou ZL, Wang LP, Yang YP, Duan YW (2020) Processes on reproductive ecology of plant species in the Qinghai-Xizang Plateau and adjacent highlands. Chinese Journal of Plant Ecology, 44, 1-21. (in Chinese with English abstract) [张婵, 安宇梦, Jäschke Y, 王林林, 周知里, 王力平, 杨永平, 段元文 (2020) 青藏高原及周边高山地区的植物繁殖生态学研究 进展. 植物生态学报, 44, 1-21.]

(责任编委：黄双全 责任编辑：黄祥忠)

\section{附录 Supplementary Material}

\section{附录1 高蔊菜各株系花粉限制分析}

Appendix 1 Pollen limitation analysis of Rorippa elata accessions

https://www.biodiversity-science.net/fileup/PDF/2021056-1.pdf 
胡正艳, 郑全晶, 母其勇, 杜志强, 刘琳, 星耀武, 韩廷申 (2021) 不同纬度高蔊菜的交配系统和繁殖保障. 生物 多样性, 29, 712-721. http://www.biodiversity-science.net/CN/10.17520/biods.2021056

附录 1 高蔊菜各株系花粉限制分析

Appendix 1 Pollen limitation analysis of Rorippa elata accessions

\begin{tabular}{lll}
\hline 株系 Accession & $\mathrm{SS}_{\text {hyb }}-\mathrm{SS}_{\text {open }}$ & $P$ \\
\hline RO794-2 & -0.204 & 0.063 \\
RO471-2 & -0.092 & 0.039 \\
RO793-5 & -0.072 & 0.046 \\
RO178-11 & -0.059 & 0.432 \\
RO468-5 & -0.054 & 0.326 \\
RO794-3 & -0.048 & 0.049 \\
RO792-4 & -0.047 & 0.284 \\
RO531-2 & -0.045 & 0.663 \\
RO531-5 & -0.039 & 0.648 \\
RO463-5 & -0.026 & 0.369 \\
RO466-4 & -0.021 & 0.710 \\
RO794-4 & -0.021 & 0.317 \\
RO456-3 & -0.012 & 0.923 \\
RO473-1 & -0.009 & 0.735 \\
RO178-10 & 0.000 & 0.995 \\
RO153-1 & 0.014 & 0.824 \\
RO178-9 & 0.014 & 0.678 \\
RO473-4 & 0.029 & 0.249 \\
RO463-2 & 0.031 & 0.553 \\
RO246-2 & 0.032 & 0.707 \\
RO810-3 & 0.047 & 0.286 \\
RO466-3 & 0.051 & 0.519 \\
RO463-3 & 0.062 & 0.159 \\
RO531-1 & $\mathbf{0 . 0 6 7}$ & $\mathbf{0 . 0 1 8}$ \\
RO468-6 & $\mathbf{0 . 0 7 3}$ & $\mathbf{0 . 0 0 6}$ \\
RO466-5 & 0.076 & 0.052 \\
RO171-3 & 0.084 & 0.100 \\
RO537-2 & $\mathbf{0 . 1 1 9}$ & $\mathbf{0 . 0 0 7}$ \\
\hline
\end{tabular}

